\title{
A phase I trial of the CDK 4/6 inhibitor palbociclib in pediatric patients with progressive brain tumors: a Pediatric Brain Tumor Consortium study (PBTC-042)
}

\author{
David Van Mater ${ }^{1}$, Sridharan Gururangan ${ }^{2}$, Oren Becher ${ }^{3}$, Olivia Campagne ${ }^{4}$, Sarah \\ Leary $^{5}$, Joanna Phillips ${ }^{6}$, Jie Huang ${ }^{7}$, Tong Lin ${ }^{7}$, Tina Young-Poussaint ${ }^{8}$, Stewart \\ Goldman $^{9}$, Patricia Baxter ${ }^{10}$, Girish Dhall ${ }^{11}$, Giles Robinson ${ }^{12}$, Mariko DeWire ${ }^{13}$, Eugene \\ Hwang $^{14}$, Clinton Stewart ${ }^{7}$, Arzu Onar ${ }^{7}$, Ira Dunkel ${ }^{15}$, and Maryam Fouladi ${ }^{16}$ \\ ${ }^{1}$ Duke University School of Medicine \\ ${ }^{2}$ Preston A. Wells Jr. Center for Brain Tumor Therapy, University of Florida \\ ${ }^{3}$ Ann and Robert H Lurie Children's Hospital of Chicago \\ ${ }^{4}$ St Jude Children's Research Hospital \\ ${ }^{5}$ Seattle Childrens Hospital \\ ${ }^{6} \mathrm{UCSF}$ \\ ${ }^{7}$ St. Jude Children's Research Hospital \\ ${ }^{8}$ Children's Hospital Boston \\ ${ }^{9}$ Ann \& Robert H Lurie Children's Hospital of Chicago \\ ${ }^{10}$ Baylor College of Medicine \\ ${ }^{11}$ Children's of Alabama \\ ${ }^{12}$ St. Jude \\ ${ }^{13}$ Cincinnati Children's Hospital Medical Center \\ ${ }^{14}$ Children's National Medical Center \\ ${ }^{15}$ Memorial Sloan Kettering Cancer Center \\ ${ }^{16}$ Children's Hospital Cincinnati
}

October 8, 2020

\begin{abstract}
Background Disruption of critical cell cycle regulators is a potential therapeutic target for brain tumors in children and adolescents. The aim of this study was to determine the maximum tolerated dose (MTD) and describe toxicities related to palbociclib, a selective cyclin dependent kinase 4/6 (CDK4/6) inhibitor in pediatric patients with progressive/refractory brain tumors with intact retinoblastoma protein. Methods Palbociclib was administered orally starting at $50 \mathrm{mg} / \mathrm{m} 2$ daily for the first 21 days of a 28 day course. Dose escalation was according to the Rolling-6 statistical design in less heavily (Stratum I) and heavily pretreated (Stratum II) patients, and MTD was determined separately for each group. Pharmacokinetic studies were performed during the first course, and pharmacodynamic studies were conducted to evaluate relationships between drug levels and toxicities. Pharmacogenetic analyses were based on pre-treatment samples. Results A total of 21 patients were enrolled on Stratum I and 14 patients on Stratum II. The MTD for both strata was $75 \mathrm{mg} / \mathrm{m} 2$. Palbociclib absorption (mean Tmax between 4.9 and 6.6 h) and elimination (mean half-life between 11.3 and $19.5 \mathrm{~h}$ ) were assessed. The most common toxicity was myelosuppression. Higher palbociclib exposure was associated with grade $3 / 4$ neutropenia and leukopenia. No patients had an objective response to palbociclib therapy. Conclusions Palbociclib was safely administered to children and adolescents at a dosage of $75 \mathrm{mg} / \mathrm{m} 2 \mathrm{for}$
\end{abstract}


21 consecutive days followed by 7 days of rest in both strata. Future studies will be required to establish its optimal utilization in pediatric patients with brain tumors.

\section{Background}

Disruption of critical cell cycle regulators is a potential therapeutic target for brain tumors in children and adolescents. The aim of this study was to determine the maximum tolerated dose (MTD) and describe toxicities related to palbociclib, a selective cyclin dependent kinase 4/6 (CDK4/6) inhibitor in pediatric patients with progressive/refractory brain tumors with intact retinoblastoma protein.

\section{Methods}

Palbociclib was administered orally starting at $50 \mathrm{mg} / \mathrm{m}^{2}$ daily for the first 21 days of a 28 day course. Dose escalation was according to the Rolling-6 statistical design in less heavily (Stratum I) and heavily pretreated (Stratum II) patients, and MTD was determined separately for each group. Pharmacokinetic studies were performed during the first course, and pharmacodynamic studies were conducted to evaluate relationships between drug levels and toxicities. Pharmacogenetic analyses were based on pre-treatment samples.

\section{Results}

A total of 21 patients were enrolled on Stratum I and 14 patients on Stratum II. The MTD for both strata was $75 \mathrm{mg} / \mathrm{m}^{2}$. Palbociclib absorption (mean $\mathrm{T}_{\max }$ between 4.9 and $6.6 \mathrm{~h}$ ) and elimination (mean half-life between 11.3 and $19.5 \mathrm{~h}$ ) were assessed. The most common toxicity was myelosuppression. Higher palbociclib exposure was associated with grade $3 / 4$ neutropenia and leukopenia. No patients had an objective response to palbociclib therapy.

\section{Conclusions}

Palbociclib was safely administered to children and adolescents at a dosage of $75 \mathrm{mg} / \mathrm{m} 2$ for 21 consecutive days followed by 7 days of rest in both strata. Future studies will be required to establish its optimal utilization in pediatric patients with brain tumors.

\section{INTRODUCTION}

Children with relapsed/refractory primary central nervous system (CNS) tumors have a dismal prognosis, and there is a critical need for novel therapeutics. Cyclin-dependent kinases (CDKs) represent an appealing target. CDKs coordinate the transition between different stages of the cell cycle, and they are regulated by cyclins. Recent studies have shown that a significant subset of pediatric CNS tumors harbor mutations in the CDK4/6 signaling axis. ${ }^{1-3}$ CDK4 and CDK6 drive the transition from the G1 growth phase to chromosomal replication in the $\mathrm{S}$ phase, and they are regulated by cyclins D1, D2, D3, and E. ${ }^{4} \mathrm{~A}$ central node in the $\mathrm{G} 1$ to $\mathrm{S}$ transition is the retinoblastoma $(\mathrm{Rb})$ protein. In an unphosphorylated state, Rb sequesters E2F transcription factors that drive cells through the S phase. Phosphorylation of Rb by CDK4/6 releases the E2F transcription factors, which results in progression of the cell cycle through $\mathrm{S}$ phase. ${ }^{4}$ This transition is very carefully regulated to prevent mutated cells from progressing through mitosis in normal cells. However, this process is corrupted in pediatric CNS tumors by a variety of mechanisms including genetic loss of $R B 1$ , amplification of CCND1, and deletion of genes encoding CDK inhibitors such asCDKN2a .

Palbociclib (IBRANCE ${ }^{\circledR}$, Pfizer Inc, Kenilworth, NJ) is an orally bioavailable, highly specific inhibitor of CDK4/6. Palbociclib exhibits pH-dependent solubility and high permeability. Palbociclib is FDAapproved for the treatment of advanced or metastatic breast cancer in combination with either fulvestrant or letrozole. ${ }^{6,7}$ Preclinical studies of palbociclib in a variety of CNS tumor cell lines and patient-derived xenograft mouse models demonstrated efficacy. ${ }^{8-11}$ Additionally, preclinical studies confirmed that palbociclib could penetrate the blood-tumor barrier and that intact $\mathrm{Rb}$ is required for its therapeutic effect. ${ }^{8}$ Here we report the results of a phase I trial assessing safety and efficacy of palbociclib in children with brain tumors (PBTC-042).

\section{PATIENTS AND METHODS}


The primary objectives of this Phase I study (PBTC-042, NCT02255461) were to determine the maximum tolerated dose (MTD)/recommended Phase II dose (RP2D), describe toxicities, and characterize palbociclib pharmacokinetics. Secondary objectives were to record preliminary evidence of palbociclib antitumor effect, evaluate CDK4/6, cyclin D1-3, Ink4a-ARF copy-number variations, explore potential relationships between palbociclib pharmacokinetics and pharmacodynamics, and relate pharmacogenetic polymorphisms to palbociclib pharmacokinetics.

Eligibility

Subjects were [?]4 years and [?]21 years of age with progressive or refractory brain tumors (except low grade gliomas) with measurable disease and Lansky or Karnofsky score [?] 60. All subjects were required to be able to swallow pills, and there were minimum body surface area restrictions at each dosing level based on available capsule strengths. Since $\mathrm{Rb}$ is required for palbociclib-mediated suppression of the CDK4/6 signaling pathway, a screening test for the presence or absence of Rb was performed in all types of brain tumors except for diffuse intrinsic pontine glioma (DIPG), ${ }^{12}$ medulloblastoma, ${ }^{13,14}$ and atypical teratoid rhabdoid tumor (ATRT), ${ }^{15}$ all of which have been shown to have $R B 1$ mutation/loss or at a very low frequency or not at all. Since myelosuppression is the main dose limiting toxicity (DLT) in adults treated with palbociclib ${ }^{6,7}$ and more heavily pretreated patients are likely to experience more severe hematologic toxicity, subjects were divided into two strata: Stratum I included patients that were not heavily pretreated, while Stratum II included heavily pretreated patients, defined as having received $>4$ prior regimens (either chemotherapy or biologic agents with myelosuppressive effects), and/or craniospinal irradiation (CSI), and/or myeloablative chemotherapy plus bone marrow or peripheral blood stem cell rescue. Subjects not meeting these criteria were eligible for Stratum I. Subjects must have had at least 3 weeks from last myelosuppressive therapy, at least 6 weeks from nitrosourea, [?]7 days from a biologic agent (at least 3 weeks if prolonged half-life), $>2$ weeks from focal irradiation, and [?]3 months from a bone marrow/stem cell infusion or CSI. Subjects were required to be on a stable dose of corticosteroids and have a stable neurological examination for at least 1 week prior to study enrollment. Subjects had to have adequate bone marrow (absolute neutrophil

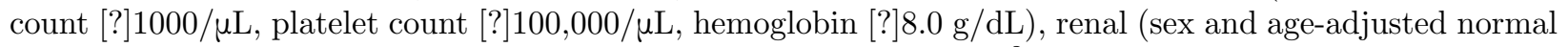
serum creatinine or glomerular filtration rate [?]70 $\mathrm{mL} / \mathrm{min} / 1.73 \mathrm{~m}^{2}$ ), and liver function (total bilirubin [?]1.5 $\times$ and alanine aminotransferase [?] $3 \mathrm{x}$ the institutional upper limit of normal for age and albumin [?]3 $\mathrm{g} / \mathrm{dL}$ ). Subjects were excluded if pregnant or lactating, cataracts noted on ophthalmologic examination, QTc $>450 \mathrm{msec}$, or prior treatment with a CDK inhibitor. Subjects of childbearing/fathering potential had to consent to birth control. Informed consent and assent were obtained according to institutional guidelines. Institutional review boards of participating institutions maintained protocol approval throughout the study.

Treatment Regimen, Drug Administration, and Dose Escalation

Palbociclib was supplied by Pfizer as 75, 100, and $125 \mathrm{mg}$ capsules, taken orally once a day for 21 days followed by a 7-day rest. One course was equivalent to 28 days. Patients were encouraged to take palbociclib with food at the same time each day. The pediatric equivalent dosage of the adult MTD (125 mg daily on this same schedule $)^{6,7}$ was approximately $75 \mathrm{mg} / \mathrm{m}^{2}$. The starting dosage for Stratum I was $50 \mathrm{mg} / \mathrm{m}^{2}$ with planned dose escalations to $75 \mathrm{mg} / \mathrm{m}^{2}$ and $95 \mathrm{mg} / \mathrm{m}^{2}$. The planned starting dosage for Stratum II was one dosage level below the MTD for Stratum I. Dosage escalation was governed by the Rolling- 6 statistical design separately in each of the two strata. ${ }^{16}$ Therapy was allowed to continue for up to two years (26 courses) in the absence of disease progression or unacceptable toxicity.

\section{Definition of MTD and DLT}

Toxicities were graded according to version 4.0 of the NCI Common Terminology Criteria for Adverse Events. A non-hematologic DLT was defined as any grade 4 non-hematologic toxicity, any grade 3 non-hematologic toxicity (except for grade 3 nausea and vomiting $<5$ days, grade 3 diarrhea or electrolyte disturbance that has not been maximally treated, or grade 3 AST/ALT elevation that resolves within 7 days and does not recur), or any grade 2 non-hematologic toxicity that persists for $>7$ days and is considered medically significant or sufficiently intolerable by patients and requires treatment interruption. A hematologic DLT 
was defined as grade 3 neutropenia with fever and sepsis, any grade 4 hematologic toxicity with the exception of lymphopenia, grade 3 thrombocytopenia, or requiring a platelet transfusion on 2 separate days in a 7 day span.

MTD was defined based on the Rolling- 6 design as the highest dose studied where no more than 1/6 patients experienced a DLT and the next higher dose level was determined to be intolerable. Patients were evaluable for MTD estimation if they received at least one dose of the study drug and were taken off treatment for toxicity during the first course (dose-finding period). In the absence of toxicity, patients needed to receive 17 or more doses of prescribed therapy during the dose-finding period to be evaluable for MTD estimation.

Definition of Response

Tumor response was defined as follows: 1) Complete response (CR), complete disappearance on MR of all enhancing tumor and mass effect. 2) Partial response (PR), [?]50\% reduction in tumor size by bi-dimensional measurement, as compared with the baseline measurements. Both CR and PR require a stable or decreasing dose of corticosteroids, accompanied by a stable or improving neurologic examination, and maintained for at least 8 weeks. If cerebrospinal fluid (CSF) was positive for malignant cells at presentation, then it must be negative on repeat assessment. 3) Stable disease (SD), neurologic exam is at least stable and maintenance corticosteroid dose not increased, and MR/CT imaging meets neither the criteria for PR nor progressive disease (PD). CSF can be positive or negative for malignant cells. 4) PD, progressive neurologic abnormalities or worsening neurologic status not explained by causes unrelated to tumor progression (e.g., anticonvulsant or corticosteroid toxicity, electrolyte disturbances, sepsis, hyperglycemia, etc.), OR a greater than $25 \%$ increase in the bi-dimensional measurement, taking as a reference the smallest disease measurement recorded since the start of protocol therapy, OR the appearance of a new lesion (including new appearance of malignant cells in the CSF), OR increasing doses of corticosteroids required to maintain stable neurological status or imaging. All eligible patients who received at least one dose of the study drug were evaluable for response assessment.

Pharmacokinetics

Pharmacokinetic studies of palbociclib after an oral dosage were performed on days 1, 2, 3, 21, and 22 of course 1. On day 2 of course 1 the palbociclib dose was held. On day 1 of course 1 , palbociclib single dose serial blood samples were drawn pre-dose and $0.5,1,2,4,8(+-1), 24(+-4)$, and $48(+-4)$ hours (immediately prior to the Day 3 dose) after the oral dose. On day 21 of course 1, palbociclib steady state serial blood samples were collected pre-dose and 1, 2, 4, $8(+-1)$, and $24(+-4)$ hours (immediately prior to the Day 22 dose) after the dose. The blood samples were collected in $\mathrm{K}_{2}$-EDTA tubes and spun to plasma within one hour of collection and stored at -20degC until analysis. Palbociclib plasma concentrations were measured by a validated liquid chromatography-mass spectrometric assay method with a lower limit of quantitation of 1 $\mathrm{ng} / \mathrm{ml}$.

Non-compartmental techniques were used to analyze the concentration-time data for palbociclib. The peak plasma concentration $\left(\mathrm{C}_{\max }\right)$ and time to $\mathrm{C}_{\max }\left(\mathrm{t}_{\max }\right)$ were determined from the plasma concentration-time profile. The last three measurable concentration-time data points in the serial sampling window were used to define the log-linear terminal slope $(\beta)$, and the terminal half-life $\left(t_{1 / 2}\right)$ was calculated as $t_{1 / 2}=\ln (2) / \beta$. The area under the plasma concentration versus time curve from time zero to the last measurable sampling time point $\left(\mathrm{AUC}_{0 \text {-Tlast }}\right)$ was calculated using the linear-up/log-down trapezoidal rule and the area under the curve from time zero to time infinity $\left(\mathrm{AUC}_{0-[?]}\right)$ was calculated by extrapolating $\mathrm{AUC}_{0 \text {-Tlast }}$ from the last measurable time point $\left(\mathrm{C}_{\text {last }}\right)$ using $\beta$ : $\left(\mathrm{AUC}_{0 \text {-Tlast }}+\mathrm{C}_{\text {last }} / \beta\right)$. The BSA-normalized apparent oral clearance $(\mathrm{CL} / \mathrm{F})$ was calculated as the BSA-normalized dose divided by $\mathrm{AUC}_{0-[?]}$.

Exposure-toxicity associations

Associations were explored between palbociclib $\mathrm{C}_{\max }$ and $\mathrm{AUC}_{0 \text {-Tlast }}$ after single and repeated doses and hematologic toxicities occurring during course 1 that were at least possibly attributable to the drug. Toxicities included neutropenia, thrombocytopenia, lymphopenia, and leukopenia. Patients were classified into three 
categories $(0 / 1 / 2)$ based on their highest toxicity grade reported for course 1 for each toxicity: $0=$ no toxicity reported, $1=$ grade 1 or 2 , and $2=$ grade 3 or 4 . Ordinal logistic regression models were built for each hematological toxicity outcome, and explanatory pharmacokinetic variables were transformed by dividing by 100. Statistical significance was defined by a p-value $P<0.05$.

Pharmacogenetics

In consenting patients, prior to the first palbociclib dose, whole blood $(5 \mathrm{~mL})$ was collected for DNA extraction with a Gentra Puregene Blood Kit (\#158389, Qiagen). DNA was quantified by using Nanodrop 2000 Spectrophotometer (Thermo Fisher Scientific). Genome-wide genotyping was performed in germline DNA with an Illumina Infinium Omni2.5Exome-8 Bead-Chip (Illumina Inc.) Selected genes included $C Y P 3 A 4$ ,CYP3A5, and SULT2A1 involved in palbociclib metabolism, and $A B C B 1, A B C G 2, A B C C 1$, and $A B C C 4$ which encode genes for known drug transporters. All available single nucleotide polymorphisms (SNPs) from these genes with a reported minor allelic frequency $>5 \%$ (https://www.ncbi.nlm.nih.gov/snp/) were extracted.

Associations between palbociclib $\mathrm{C}_{\max }$ and $\mathrm{AUC}_{0 \text {-Tlast }}$ after single and repeated doses and genotypes were evaluated using non-parametric Kruskal-Wallis tests, Mann-Whitney U-tests, and linear regression. For SNPs including data from wild-type (0), heterozygous (1), and homozygous mutant (2) patients, the distribution of the pharmacokinetic variables was compared between 0,1 , and 2 , and between $0+1$ vs 2 , and 0 vs $1+2$. Statistical significance was defined by a p-value $P<0.05$. Strong linkage disequilibrium (LD) between SNPs, defined as $\mathrm{r}^{2}>0.80$, was verified using the LD matrix tool (https://ldlink.nci.nih.gov/ ). Based on the population size, associations were tested using a univariate analysis only. All the tests were performed using $\mathrm{R}^{\circledR}$ software.

Immunohistochemistry for $\mathrm{Rb}$

Formalin fixed paraffin embedded tumor tissue were collected from all patients prior to enrollment (except for those with DIPG, medulloblastoma, or ATRT). Immunohistochemistry for Rb was performed as previously described $^{17}$ using a mouse monoclonal anti-Rb antibody (G3-245; BD Biosciences, San Jose, CA) and an automated IHC staining process (Benchmark XT; Ventana Medical Systems, Inc, Tucson, AZ). Briefly, antigen retrieval was performed in Tris, $\mathrm{pH} 8.0$ at $95 \mathrm{C}$ for 1 hour, followed by incubation in $3 \% \mathrm{H}_{2} \mathrm{O}_{2}$ for $16 \mathrm{~min}$, and primary antibody at 1:100 at room temperature for 60 minutes. Tumor Rb1 protein status was denoted as "positive" if [?]20\% of tumor cells had positive nuclear staining. Rb1-positive endothelial cells served as an internal positive control. All slides were centrally reviewed by a dedicated neuropathologist (JJP).

\section{RESULTS:}

\section{Subject characteristics}

In Stratum I, a total of 21 patients were enrolled. A total of 28 patients were pre-screened with $22 \mathrm{Rb}-$ positive, 5 Rb-negative, and 1 with inadequate tissue. Of the 22 patients with Rb-positive tumors, 14 elected to enroll for treatment. The remaining 7 patients had DIPG and did not require Rb testing (Table 1 ). For Stratum II, 14 patients were enrolled. A total of 15 patients were pre-screened with 12 Rb-positive and $3 \mathrm{Rb}$-negative. Of the 12 patients with Rb-positive tumors, 9 elected to enroll for treatment. The remaining 5 patients had DIPG or medulloblastoma and did not require Rb testing (Table 1 ). The most common tumor type was DIPG for stratum I (7/21 patients) and ependymoma for stratum II (7/14 patients). There was a male predominance in Stratum II (Table 1 ).

\section{Toxicities}

DLTs are summarized in Table 2 . Three dosage levels were assessed in Stratum I. At dosage level 1 (50 $\left.\mathrm{mg} / \mathrm{m}^{2}\right), 0 / 3$ patients experienced a DLT. At dosage level $2\left(75 \mathrm{mg} / \mathrm{m}^{2}\right)$, there were no DLTs in the first 3 patients, prompting dose escalation. At dosage level $3\left(95 \mathrm{mg} / \mathrm{m}^{2}\right)$, there were 6 patients enrolled, 4 of which were evaluable (1 patient received less than the required amount of study agent due to progressive disease 
and 1 patient voluntarily withdrew after initiating protocol therapy). Two of the 4 patients developed grade 4 neutropenia. Hence the MTD was exceeded at dosage level 3, and a total of 9 additional patients were enrolled at dosage level 2. An MTD of $75 \mathrm{mg} / \mathrm{m}^{2}$ was established for Stratum I, with only 2/12 patients experiencing a DLT (Table 2 ).

We proceeded to Stratum II after establishing a MTD for Stratum I. Given concern for myelosuppression in the heavily pretreated patients, enrollment in Stratum II was started at $50 \mathrm{mg} / \mathrm{m}^{2}$ (1 dose level below the MTD of Stratum I). Four patients were enrolled at $50 \mathrm{mg} / \mathrm{m}^{2}$, all were evaluable with no DLTs. This prompted a dose increase to $75 \mathrm{mg} / \mathrm{m}^{2}$ where a total of 10 subjects were enrolled and 7 were evaluable for DLT assessment (2 patients progressed before receiving sufficient study drug and 1 patient withdrew prior to treatment initiation). No DLT was observed among the first 6 evaluable subjects. There was no intent of escalating beyond the MTD of Stratum I, so the cohort was expanded to include a total of 12 patients. However, slow accrual prompted closure of the study prior to completing enrollment. One of 7 evaluable patients experienced a DLT, establishing $75 \mathrm{mg} / \mathrm{m}^{2}$ as the MTD for both Stratum I and II.

Adverse events associated with palbociclib in Stratum I and II are outlined in Table $\mathbf{3}$ and Table 4 , respectively. Similar to the side effect profile in adults, ${ }^{18}$ the most common adverse events were related to myelosuppression with decrease in white blood cells, neutrophils, lymphocytes, and platelets being the most common.

\section{Treatment response}

No patients on either stratum had an objective response to palbociclib. A patient with anaplastic ependymoma in Stratum I was treated on a dose of $75 \mathrm{mg} / \mathrm{m}^{2}$ for 18 courses before coming off therapy. This patient was a 21 year-old male with neurofibromatosis type I and a multiply recurrent WHO grade III anaplastic ependymoma first diagnosed when he was 14 years-old. Prior treatment included multiple surgeries and 2 rounds of radiation therapy. He had 7 courses of 5 -fluorouracil followed by a 1.5 year off-therapy period before starting palbociclib. Subsequent to his progression on palbociclib, he was maintained on additional chemotherapy regimens for 2.5 years before dying of his tumor. A mutation profile of his tumor confirmed a C11orf95-RELA fusion (pathognomonic of ependymoma), alterations in NF1, and homozygous deletion of $C D K N 2 A$ and $C D K N 2 B$. He additionally had multiple segmental chromosomal gains and losses across the genome. Another patient with a GBM treated at $75 \mathrm{mg} / \mathrm{m}^{2}$ stayed on treatment for 6 courses. In Stratum II, 3 subjects (2 at $50 \mathrm{mg} / \mathrm{m}^{2}$ and 1 at $75 \mathrm{mg} / \mathrm{m}^{2}$ ) remained on treatment for 4 courses. In Stratum I and II, $16 / 21(76 \%)$ and $12 / 13(92 \%)$ of patients, respectively, who initiated protocol therapy experienced disease progression while on treatment. The majority of patients received 2 or fewer cycles of therapy (Table 5 )

\section{Pharmacokinetics}

Pharmacokinetic data after single dose palbociclib were collected and analyzed for a total of 34 patients $(7$ at $50 \mathrm{mg} / \mathrm{m}^{2}, 21$ at $75 \mathrm{mg} / \mathrm{m}^{2}$, and 6 at $95 \mathrm{mg} / \mathrm{m}^{2}$ ). Data after repeated palbociclib doses were available for a total of 27 patients $\left(6\right.$ at $50 \mathrm{mg} / \mathrm{m}^{2}, 18$ at $75 \mathrm{mg} / \mathrm{m}^{2}$, and 3 at $95 \mathrm{mg} / \mathrm{m}^{2}$ ). The palbociclib plasma concentration-time profiles are depicted for each dosing group in Supplementary Figure S1, and the associated pharmacokinetic parameters are reported in Table 6 .

Palbociclib was absorbed with a mean $\mathrm{T}_{\max }$ between 4.9 and $6.6 \mathrm{~h}$ post-dose, and exhibited an elimination with a mean half-life between 11.3 and $19.5 \mathrm{~h}$ and a mean apparent oral clearance ranging from 26.8 and $52.6 \mathrm{~L} / \mathrm{h} / \mathrm{m}^{2}$. Within 50 and $95 \mathrm{mg} / \mathrm{m}^{2}$, following single and repeated doses, mean palbociclib $\mathrm{C}_{\max }$ and $\mathrm{AUC}_{0 \text {-Tlast }}$ increased in proportional manner; however, large inter-individual variabilities were observed among the pharmacokinetic parameters.

Exposure-toxicity associations

Associations were found between palbociclib steady state pharmacokinetic variables and neutropenia. Higher values of palbociclib steady state $\mathrm{C}_{\max }$ and $\mathrm{AUC}_{0-24 \mathrm{~h}}$ were associated with severe (grade 3 or 4 ) neutropenia ( $\mathrm{p}$-values $=0.014$ and 0.009 , respectively). The odds ratio estimates (95\% Wald confidence) were 7.49 (1.50-37.46) and 1.16 (1.04-1.30), respectively. In addition, associations were found between palbociclib 
pharmacokinetic variables and leukopenia. Higher values of single-dose palbociclib $\mathrm{AUC}_{0-48 \mathrm{~h}}$, and steady state $\mathrm{C}_{\max }$ and $\mathrm{AUC}_{0-24 \mathrm{~h}}$ were associated with severe leukopenia (p-values $=0.038,0.044$ and 0.036 , respectively). The odds ratio estimates were 1.10 (1.01-1.20), 3.95 (1.04-15.0) and 1.10 (1.01-1.19), respectively. No association was found between palbociclib pharmacokinetic variables and thrombocytopenia or lymphopenia.

Pharmacogenetics

DNA was collected from 32 patients and a total of 795 SNPs ( 8 for $C Y P 3 A 4,7$ for $C Y P 3 A 5,16$ for SULT2A1 , 110 for $A B C B 1$, 85 for $A B C G 2,179$ for $A B C C 1$ and 390 for $A B C C 4$ ) were extracted from the array. Significant associations between genotypes and palbociclib $\mathrm{C}_{\max }$ and $\mathrm{AUC}_{0 \text {-Tlast }}$ following single $(\mathrm{N}=32)$ and repeated $(\mathrm{N}=24)$ doses are reported in Supplementary Tables S1-4 andFigures S2-5. The frequency of significant SNPs is reported inSupplementary Table S5 . Multiples associations were found between palbociclib pharmacokinetic variables and SNPs fromSULT2A1, ABCB1 , ABCG2 , ABCC1, and $A B C C 4$; however, no associations were found with SNPs from $C Y P 3 A 4$ and $C Y P 3 A 5$ genes.

\section{DISCUSSION}

There is a critical need for novel agents to treat refractory/progressive brain tumors in children and adolescents. Palbociclib represents an intriguing candidate as it is a highly specific CDK4/6 inhibitor that is already FDA approved for adult breast cancer patients. This was the first study to test the safety and tolerability of palbociclib in pediatric patients. The MTD was $75 \mathrm{mg} / \mathrm{m}^{2}$ for Stratum I and II patients. Myelosuppression was the primary toxicity, among which grade 3/4 neutropenia and leukopenia were found to be significantly associated with higher palbociclib exposure. A similar exposure-toxicity association was also characterized in adult patients. ${ }^{19}$

No previous pharmacokinetic parameters have been reported for palbociclib in children with cancer. In this patient population, palbociclib displayed a similar pharmacokinetic profile as seen in healthy adults and adults with solid tumors. ${ }^{20,21}$ Palbociclib $\mathrm{T}_{\max }, \mathrm{C}_{\max }$, and $\mathrm{AUC}_{0 \text {-Tlast }}$ observed in this study were similar to the values previously reported in adults following 100 and $125 \mathrm{mg}$ palbociclib doses. ${ }^{20}$ The mean half-life $(\sim 15.6 \mathrm{~h})$ observed in this study was slightly lower that reported in adults (23-26 h); however this might be explained by the shorter sampling design used in this trial. ${ }^{20} \mathrm{~A}$ population-based pharmacokinetic analysis will be performed to further characterize the disposition of palbociclib in this population, quantify the inter-individual variability, and determine the potential influence of patient covariates.

Although palbociclib has been confirmed in vitro and in vivo to be a substrate for $C Y P 3 A$ genes, ${ }^{22-24}$ no associations were found between palbociclib pharmacokinetics and variants from CYP3A4 and CYP3A5 genes in our pediatric population. Multiple associations were found with SULT2A1 gene which is predominantly involved in palbociclib sulfonation, ${ }^{23}$ and with genes coding for known drug transporters. Due to the small sample size, no multivariate analysis was performed, and p-values were not adjusted for multiplicity. Thus, these associations will need to be confirmed by further studies.

Only a few patients proceeded beyond 2 courses of therapy, and no patients had an objective response to palbociclib on this study. A similar lack of efficacy was reported in a recent phase 2 study of adult patients with recurrent, Rb-positive glioblastoma. ${ }^{25}$ Notably, a subset of these patients were treated with palbociclib prior to surgery, and the tumor concentration of palbociclib was greater than the $0.06 \mu \mathrm{M}$ concentration felt to be biologically effective. ${ }^{25}$ Thus palbociclib was able to cross the brain-tumor barrier in selected patients. The authors concluded that palbociclib is not likely to be effective as monotherapy in pretreated patients, but it may have a role earlier in therapy in patients with distinct mutational events or in combination with radiation or other biological agents. ${ }^{25}$ There are ongoing clinical trials in adult breast cancer patients with CNS metastases to better elucidate the role of palbociclib and other CDK4/6 inhibitors in that clinical context; data to-date are sparse. ${ }^{26}$

CDK4/6 is central to cell cycle control in cells with intact Rb, but enhanced vulnerability to CDK4/6 inhibition may be seen in tumors with genetic mutations that increase signaling though the cyclin D-CDK4/6$\mathrm{Rb}$ pathway. Examples include amplification of $C C N D 1$, CCND2 ,CCND3 , CDK4, or CDK6 , or deletion 
of $C D K N 2 A$. One might expect enhanced efficacy of palbociclib in a patient population enriched with tumors harboring such mutations. The National Cancer Institute-Children's Oncology Group Pediatric MATCH Screening Trial (NCT NCT03155620) is conducting such a study for refractory pediatric tumors in a tissueagnostic fashion. The MTD of palbociclib in this study will inform the dose for the MATCH study and similar studies in the future.

Additionally, there is a growing recognition that CDK4/6 inhibitors such as palbociclib are maximally effective in combination with other agents. ${ }^{27}$ Notably, palbociclib was FDA-approved in combination with anti-estrogen agents for the treatment of patients with breast cancer patients. ${ }^{6,7}$ Preclinical studies are required to elucidate palbociclib resistance mechanisms that can exploited in individual cancer types. For example, $\mathrm{mTOR}^{28}$ and c-Met/ $\mathrm{Trk}^{29}$ represent intriguing targets for intervention in glioblastoma.

In summary, we describe the MTD, toxicity, pharmacokinetic and pharmacogenomic data for palbociclib in children and adolescents. There was no notable antitumor activity efficacy seen for progressive/refractory brain tumors in this trial, but the cyclin D-CDK4/6-Rb pathway remains an intriguing target for future investigations. Future studies will strive to identify subsets of patients with enhanced vulnerability to CDK4/6 inhibition and agents that can be combined with palbociclib for synergy.

\section{CONFLICT OF INTEREST}

I.D. declares advisory/consulting roles for Roche, Apexigen, AstraZeneca, Bristol Myers Squibb/Celgene, and Fennec. A.O-T. reports advisory roles for Roche and Lilly.

\section{ACKNOWLEDGEMENTS}

The trial was partially funded by a National Cancer Institute (NCI) Cancer Therapy Evaluation Program (CTEP) PBTC U01 Grant: 2UM1CA081457 (UM1). The content is solely the responsibility of the authors and does not necessarily represent the official views of the National Institutes of Health. Partial funding was also provided by MSKCC Core Grant (P30 CA008748) and by the American Lebanese Syrian Associated Charities (ALSAC) which provides funding and infrastructure support for the PBTC Operations Core personnel. Pfizer provided Palbociclib and financial support for trial conduct, patient research costs, operations costs and correlative studies. Pfizer reviewed the manuscript but did not have a direct role in trial design, patient recruitment, data collection, analyses or manuscript preparation.

\section{REFERENCES}

1. Li M, Lockwood W, Zielenska M, et al. Multiple CDK/CYCLIND genes are amplified in medulloblastoma and supratentorial primitive neuroectodermal brain tumor. Cancer Genet. 2012;205(5):220-231.

2. Dubuc AM, Northcott PA, Mack S, Witt H, Pfister S, Taylor MD. The genetics of pediatric brain tumors. Curr Neurol Neurosci Rep.2010;10(3):215-223.

3. Gajjar A, Bowers DC, Karajannis MA, Leary S, Witt H, Gottardo NG. Pediatric Brain Tumors: Innovative Genomic Information Is Transforming the Diagnostic and Clinical Landscape. J Clin Oncol.2015;33(27):29862998.

4. Ivanchuk SM, Rutka JT. The cell cycle: accelerators, brakes, and checkpoints. Neurosurgery. 2004;54(3):692-699; discussion 699-700.

5. Paugh BS, Broniscer A, Qu C, et al. Genome-wide analyses identify recurrent amplifications of receptor tyrosine kinases and cell-cycle regulatory genes in diffuse intrinsic pontine glioma. J Clin Oncol. 2011;29(30):3999-4006.

6. Turner NC, Ro J, Andre F, et al. Palbociclib in Hormone-Receptor-Positive Advanced Breast Cancer. $N$ Engl J Med.2015;373(3):209-219.

7. Finn RS, Martin M, Rugo HS, et al. Palbociclib and Letrozole in Advanced Breast Cancer. N Engl J Med. 2016;375(20):1925-1936. 
8. Michaud K, Solomon DA, Oermann E, et al. Pharmacologic inhibition of cyclin-dependent kinases 4 and 6 arrests the growth of glioblastoma multiforme intracranial xenografts. Cancer Res.2010;70(8):3228-3238.

9. Huillard E, Hashizume R, Phillips JJ, et al. Cooperative interactions of BRAFV600E kinase and CDKN2A locus deficiency in pediatric malignant astrocytoma as a basis for rational therapy. Proc Natl Acad Sci US A. 2012;109(22):8710-8715.

10. Aoki Y, Hashizume R, Ozawa T, et al. An experimental xenograft mouse model of diffuse pontine glioma designed for therapeutic testing.J Neurooncol. 2012;108(1):29-35.

11. Cook Sangar ML, Genovesi LA, Nakamoto MW, et al. Inhibition of CDK4/6 by Palbociclib Significantly Extends Survival in Medulloblastoma Patient-Derived Xenograft Mouse Models. Clin Cancer Res.2017;23(19):5802-5813.

12. Mackay A, Burford A, Carvalho D, et al. Integrated Molecular Meta-Analysis of 1,000 Pediatric HighGrade and Diffuse Intrinsic Pontine Glioma. Cancer Cell. 2017;32(4):520-537 e525.

13. Parsons DW, Li M, Zhang X, et al. The genetic landscape of the childhood cancer medulloblastoma. Science.2011;331(6016):435-439.

14. Jones DT, Jager N, Kool M, et al. Dissecting the genomic complexity underlying medulloblastoma. Nature. 2012;488(7409):100-105.

15. Kieran MW, Roberts CW, Chi SN, et al. Absence of oncogenic canonical pathway mutations in aggressive pediatric rhabdoid tumors. Pediatr Blood Cancer. 2012;59(7):1155-1157.

16. Skolnik JM, Barrett JS, Jayaraman B, Patel D, Adamson PC. Shortening the timeline of pediatric phase I trials: the rolling six design.J Clin Oncol. 2008;26(2):190-195.

17. Goldhoff P, Clarke J, Smirnov I, et al. Clinical stratification of glioblastoma based on alterations in retinoblastoma tumor suppressor protein (RB1) and association with the proneural subtype. J Neuropathol Exp Neurol. 2012;71(1):83-89.

18. Finn RS, Crown JP, Lang I, et al. The cyclin-dependent kinase $4 / 6$ inhibitor palbociclib in combination with letrozole versus letrozole alone as first-line treatment of oestrogen receptor-positive, HER2-negative, advanced breast cancer (PALOMA-1/TRIO-18): a randomised phase 2 study. Lancet Oncol. 2015;16(1):2535.

19. Sun W, O'Dwyer PJ, Finn RS, et al. Characterization of Neutropenia in Advanced Cancer Patients Following Palbociclib Treatment Using a Population Pharmacokinetic-Pharmacodynamic Modeling and Simulation Approach. J Clin Pharmacol. 2017;57(9):1159-1173.

20. Flaherty KT, Lorusso PM, Demichele A, et al. Phase I, dose-escalation trial of the oral cyclin-dependent kinase 4/6 inhibitor PD 0332991, administered using a 21-day schedule in patients with advanced cancer. Clin Cancer Res. 2012;18(2):568-576.

21. Sun W, Wang DD. A population pharmacokinetic (PK) analysis of palbociclib (PD-0332991) in patients (pts) with advanced solid tumors. ABSTRACT. Annals of Oncology 25. 2014;(Supplement 4):v146-iv164.

22. Yu Y, Loi CM, Hoffman J, Wang D. Physiologically Based Pharmacokinetic Modeling of Palbociclib. $J$ Clin Pharmacol.2017;57(2):173-184.

23. Dhillon S. Palbociclib: first global approval. Drugs.2015;75(5):543-551.

24. Hoffman JT, Plotka A, O'Gorman M, et al. A phase 1 randomized, openlabel, fixed-sequence, 2-period study of the effect of multiple doses of rifampin on palbociclib (PD-0332991) pharmacokinetics in healthy volunteers. Cancer Research. 2015;75.

25. Taylor JW, Parikh M, Phillips JJ, et al. Phase-2 trial of palbociclib in adult patients with recurrent RB1-positive glioblastoma.J Neurooncol. 2018;140(2):477-483. 
26. Nguyen LV, Searle K, Jerzak KJ. Central nervous system-specific efficacy of CDK4/6 inhibitors in randomized controlled trials for metastatic breast cancer. Oncotarget. 2019;10(59):6317-6322.

27. Klein ME, Kovatcheva M, Davis LE, Tap WD, Koff A. CDK4/6 Inhibitors: The Mechanism of Action May Not Be as Simple as Once Thought.Cancer Cell. 2018;34(1):9-20.

28. Olmez I, Brenneman B, Xiao AZ, et al. Combined CDK4/6 and mTOR Inhibition Is Synergistic against Glioblastoma via Multiple Mechanisms. Clinical Cancer Research. 2017;23(22):6958-6968.

29. Olmez I, Zhang Y, Manigat L, et al. Combined c-Met/Trk Inhibition Overcomes Resistance to CDK4/6 Inhibitors in Glioblastoma. Cancer Research. 2018;78(15):4360-4369.

\section{Hosted file}

Tables 1-6.pdf available at https://authorea.com/users/365122/articles/485356-a-phase-itrial-of-the-cdk-4-6-inhibitor-palbociclib-in-pediatric-patients-with-progressive-braintumors-a-pediatric-brain-tumor-consortium-study-pbtc-042 